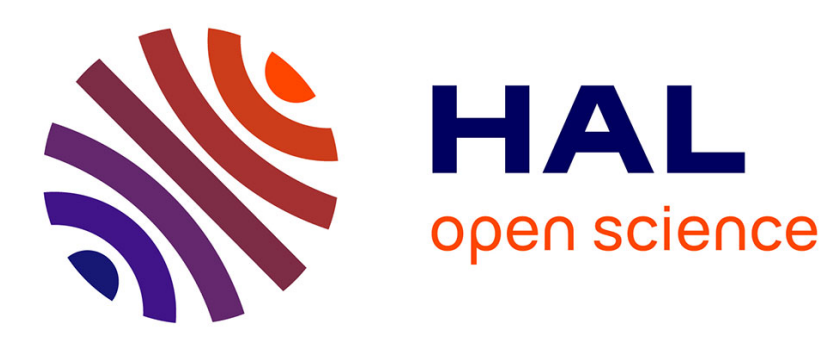

\title{
Potential of Particle Swarm Optimization and Genetic Algorithms for FIR Filter Design
}

Kamal Boudjelaba, Frédéric Ros, Djamel Chikouche

\section{To cite this version:}

Kamal Boudjelaba, Frédéric Ros, Djamel Chikouche. Potential of Particle Swarm Optimization and Genetic Algorithms for FIR Filter Design. Circuits, Systems, and Signal Processing, 2014, 33 (10), pp.3195 - 3222. 10.1007/s00034-014-9800-y . hal-01076894

\section{HAL Id: hal-01076894 \\ https://hal.science/hal-01076894}

Submitted on 23 Oct 2014

HAL is a multi-disciplinary open access archive for the deposit and dissemination of scientific research documents, whether they are published or not. The documents may come from teaching and research institutions in France or abroad, or from public or private research centers.
L'archive ouverte pluridisciplinaire HAL, est destinée au dépôt et à la diffusion de documents scientifiques de niveau recherche, publiés ou non, émanant des établissements d'enseignement et de recherche français ou étrangers, des laboratoires publics ou privés. 


\title{
Potential of Particle Swarm Optimization and Genetic Algorithms for FIR Filter Design
}

\author{
Kamal Boudjelaba ${ }^{1}$, Frédéric Ros ${ }^{1}$, Djamel Chikouche ${ }^{2}$ \\ e-mail: kamal.boudjelaba@univ-orleans.fr, frederic.ros@univ-orleans.fr,dj_chikou@yahoo.fr
}

1. Prisme Laboratory, IRAuS Pole, Axis Image and vision, Polytech'Orléans, 12 rue de Blois, Orléans, France

2. Electronics Department, University of M'sila, Rue Ichebilia, M'sila, Algeria

\begin{abstract}
This article studies the performance of two metaheuristics, Particle Swarm Optimization (PSO) and Genetic Algorithms (GA), for FIR filter design. The two approaches aim to find a solution to a given objective function but employ different strategies and computational effort to do so. PSO is a more recent heuristic search method than GA; its dynamics exploit the collaborative behavior of biological populations. Some researchers advocate the superiority of PSO over GA and highlight its capacity to solve complex problems thanks to its ease of implementation. In this paper different versions of PSOs and GAs including our specific GA scheme are compared for FIR filter design. PSO generally outperforms standard GAs in some performance criteria but our AGA (Adaptive Genetic Algorithm) is shown to be better on all criteria except CPU runtime. The study also underlines the importance of introducing intelligence in metaheuristics to make them more efficient by embedding self-tuning strategies. Furthermore, it establishes the potential complementarity of the approaches when solving this optimization problem.
\end{abstract}

Keywords FIR filters · Genetic Algorithm · Particle Swarm Optimization · Evolutionary Optimization

\section{Introduction}

In recent years, with the rapid improvement in computer technology, digital signal processing has become increasingly widespread. As a result, the design of digital filters has been receiving a great deal of attention. Digital filters can be classified into two groups, i.e., finite impulse response (FIR) filters and infinite impulse response (IIR) filters. Since FIR digital filters are inherently stable and can have linear phase, they are often preferred over IIR filters [1]. These FIR filters have many important applications, e.g., in radar and sonar signal processing and in image processing. Filter design is the process including synthesis and implementation whereby the resulting filter meets the given constraints (amplitude response, phase, etc.) [2]. From a mathematical point of view, the problem of filter design can be seen as a constrained minimization problem consisting in finding a realization of the filter which meets each of the requirements to a sufficient degree to make it useful.

There are different methods to find the coefficients from frequency specifications: Window design, Frequency Sampling, Weighted Least Squares design, or Equiripple design. The possible drawback to filters designed this way is that they contain many small ripples in the pass band and stop band, since such a filter minimizes the peak error. In the Window and Frequency Sampling methods of FIR filter design, it remains difficult to accurately control the cutoff frequencies of the pass band and stop band. The complexity of the design reaches the limits of conventional design approaches that prove unable to handle all the constraints of filter performances. While the science of digital filter design is well established, there are no conventional design procedures that lead to an optimal design. For the above reasons, the design of FIR filters is an intensively-researched area, aiming at obtaining more general and innovative techniques that are able to tackle new and complex engineering problems of great relevance today.

Several papers address the limitations of conventional approaches and emphasize the advantages of several modern heuristic tools that have evolved in the last two decades such as evolutionary computation, simulated annealing, tabu search, particle swarm, etc. These tools facilitate the solving of optimization problems that were previously difficult or impossible to tackle. Genetic algorithms (GA) are probably the most popular and the most widely 
developed metaheuristic as many alternative versions exist. They were initially proposed by Holland as an algorithm for probabilistic search, learning, and optimization, and are based in part on the mechanism of biological evolution and Darwin's theory of evolution [3]. The core of a GA algorithm repeatedly propagates generations for a large population by applying three operators, namely selection, crossover and mutation in order to balance space exploration and exploitation. More recently, particle swarm optimization (PSO) techniques (Kennedy and Eberhart, 1995) [4] have attracted considerable attention. PSO is a new branch of population-based heuristic algorithms. The particle swarm concept was motivated by the simulation of the social behavior of biological organisms. The original intent was to simulate graphically the graceful but unpredictable movement of flocks of birds. PSO is gaining more and more attention due to its fast convergence rate, simple algorithmic structure and strong global optimization capability. It has been applied to many real-world problems including clustering [510]; GA and PSO algorithms each have their respective advantages and disadvantages since both approaches aim to find a solution to a given objective function but employ different strategies and mechanisms to do so. Furthermore, it should be mentioned that there are a few weak points associated with metaheuristic methods, such as the nonexistence of theoretical proof of convergence to a global optimum in sufficiently general conditions [11]. While these drawbacks are generally not foregrounded in published work, applying these algorithms for a dedicated problem remains difficult for practitioners. For filter design, no single algorithm can be considered to solve all types of problem with the same accuracy. The space of possible filters is very large, and individual parameters are tightly coupled. Researchers sometimes use hybrid algorithms (e.g., GA and SA, PSO and ACO) or more adaptive procedures to compensate for these weaknesses [12, 13]. Especially, in [12], the PSO algorithm has been successfully applied to design a higher-order digital differentiator that contains two different structures of even and odd orders. A survey of hybridization schemes between PSO and other techniques can be found in [14].To the best of our knowledge, there are no popular hybrid schemes involving both GA and PSO methods in the field of filter design.

Several recent papers on PSOs have tended to stress their advantages rather than their weaknesses, claiming the superiority of PSOs over GAs due to their capacity to solve complex problems via an easier implementation. For the design of FIR filters, several researchers [15-19] have conducted interesting studies and performed comparisons between GAs and PSO. Their conclusion is that PSO is better than GA for the synthesis of 1-D FIR filters. It is true that compared with GAs, PSO does not need operators such as crossover and mutation that can appear complex. It also requires only primitive and simple mathematical operators. Furthermore, it is computationally inexpensive in terms of both memory and runtime especially in its primary version.

When comparing the performance of PSO and GA, it is necessary however to clearly delineate the scope of the comparison in order to deliver the real potential of these tools to practitioners. While the inspiration for these two metaheuristics is not the same, the foundations of the derived algorithms are in fact rather similar. Both of them aim to optimally explore a search space via diversification mechanisms while trying to exploit the benefits of the exploration more locally. Exploration and exploitation are managed via a process combining stochastic and deterministic tasks. Moreover, they both face the issue of parameter setting which is shared by all heuristic schemes, and both encounter problems related to premature convergence or stagnation. Their differences hinge more on the strategies used and on how mechanisms are implemented: GAs focus more on approaches derived from elitism while PSO dynamics are inspired by collaborative behavior.

Thus, from our extensive experience in GA design, we claim that the real challenge today for applying these methods in industrial contexts lies not in the search for new concepts or theories but in how existing mechanisms can be adaptively embedded to make these tools operational for non-specialist users. Choosing the right values before (or during) a run and appropriately varying parameter settings are the main challenges. Expertise and experience are not sufficient.

We therefore believe that the recent results highlighting the superiority of new PSO methods over genetic algorithms should be interpreted with much more caution. A careful review of the literature shows that most of the comparisons are limited to specific algorithms launched in specific concepts. In the filter design literature, they often concern an improved PSO version and rather more basic GAs and hence the results cannot be generalized. The principal issue for evolutionary algorithm designers is the fact that design details, i.e., parameter values, strongly influence the performance of the algorithm. By adding more or less adaptability in parameters or changing some settings, opposite conclusions can easily be reached. 
This paper is dedicated to FIR design by PSOs and GAs. The goal is to show that the performances of these tools are related more to the potential of the algorithms to be flexible or even self-tuning than to the inspiration behind the mechanisms themselves. Three PSO algorithms are compared to two versions of GAs. In addition to a basic version, we have selected two recent PSO algorithms that have proved to be efficient for filter design and that have been advantageously compared to GAs. Concerning the two GA versions, the first one called GA is a degraded version of the second one, "AGA" (Adaptive Genetic Algorithm). AGA is a proprietary version especially developed for filter design involving pre-calibrated parameters and self-tuning procedures. Based on our knowhow and a comprehensive compilation of the specialized evolutionary literature, our AGA integrates several dedicated mechanisms to assist the GA convergence for a more efficient design and to enable practical use by nonexperts with different configurations (low pass, high pass, band pass and band cut).

The comparison between techniques is important for practitioners and more generally for the field of filter design that awaits more efficient filters (in terms of accuracy, adaptability and cost reduction). The goal of this paper is also to provide some insights into how GAs and PSO work in order to exploit in the most effective way the singularity and complementarity in relevant hybrid schemes, i.e. to provide users with stable and relevant solutions without their being faced with all the evolutionary computation complexity.

The paper is organized as follows: section 2 introduces briefly the theoretical framework describing the filter design problem. In section 3 a literature review concerning GAs and PSO is presented and sub-section 3.4 is devoted to our adaptive GA. Design examples to illustrate the effectiveness of the different approaches are included in Section 4 and the results obtained in each of the proposed approaches are summarized and discussed. Some conclusions are also provided at the end.

\section{FIR filter design}

The transform function of a digital FIR filter can be characterized by

$H(z)=\sum_{n=0}^{2 N} h(n) * Z^{-n}$

where $2 N$ is the order of the filter which has $2 N+1$ coefficients. $h(n)$ is the filter impulse response calculated in the design process.

The frequency response can be expressed from the transfer function by computing its values for $Z=e^{j \omega}$.

$\widehat{H}(\omega)=\sum_{n=0}^{2 N} h(n) * e^{-j n \omega}$

In order to make the phase characteristic of an FIR filter linear, the impulse response must be symmetric, which can be expressed in the following way:

$h(n)=h(N-n-1)$

The frequency response of a linear-phase symmetric FIR digital filter with length $2 N+1$ can be characterized by

$\widehat{H}(w)=e^{-j N w} \sum_{n=0}^{N} b_{n} * \cos (n \omega)=e^{-j N w} H(\omega)$

$H(\omega)=\sum_{n=0}^{N} b_{n} * \cos (n \omega)$

where 
$b_{n}= \begin{cases}h(N) & n=0 \\ 2 h(N-n) & n=1,2, \ldots, N\end{cases}$

and $H(\omega)$ is the magnitude response which is used to approximate the desired magnitude response $D(\omega)$ in the least squares sense. For a low pass filter, the desired magnitude response can be expressed as follows:

$D(\omega)=\left\{\begin{array}{cc}1 & 0 \leq \omega \leq \omega_{c} \\ 0 & \omega>\omega_{c}\end{array}\right.$

where $\omega_{c}$ is the frequency cutoff.

Consequently, given the desired amplitude specifications $D(\omega)$ and the length of the filter $2 N+1$, the design of an FIR filter consists in searching for the optimum filter coefficients $b_{n}$ so that the desired specifications are satisfied. The error term to be minimized is the squared error between the frequency response of the ideal filter and the actual approximate filter designed.

In order to evaluate the chromosomes representing possible FIR filters, the least squared (LS) error is used to evaluate the particle.

$E(\omega)=\sum_{\omega}(W(\omega)[D(\omega)-H(\omega)])^{2}$

$W(\omega)$ is the weighting function used to weight the error differently in different frequency bands, $D(\omega)$ is the desired filter magnitude and $H(\omega)$ is the magnitude response of the designed filter.

Equation 6 can be rewritten as follows:

$E(\omega)=\sum_{\omega}\left(W(\omega)\left[D(\omega)-\sum_{n=0}^{N} b_{n} * \cos (n \omega)\right]\right)^{2}$

where $b_{n}(\mathrm{n}=0, \ldots, \mathrm{N})$ is $\mathrm{n}$-th filter coefficient.

A chromosome solution is then directly represented by a vector of coefficients representing the filter itself. It means that for a filter of $\mathrm{N}+1$ dimension, it is necessary to determine $\mathrm{N}+1$ coefficients, the goal being to find them in order to minimize the sum-squared error E over $\mathrm{m} 1$ (=128) frequency points. Chromosomes with lower errors will have a higher probability of being selected as parents.

$F=\frac{1}{1+E}$

$F$ represents the fitness function to be maximized using the genetic algorithm (GA) and particle swarm optimization (PSO).

\section{Review of GA and PSO techniques}

\subsection{Genetic Algorithm}

GAs are population-based heuristic methods, which start from an initial population of random solutions for a given problem. The core GA approach is as follows: each chromosome is coded (real coded, binary...) to be a candidate solution. It is evaluated according to a fitness function. After evaluation, there is a selection phase in which possibly good chromosomes will be chosen by a selection operator to undergo the recombination process.

'Roulette wheel' and 'Tournament' selection are the most popular operators for selecting chromosomes. Selection pressure or elitism favors the partial or full reproduction of the best chromosomes [20, 21]. In the recombination phase, crossover and mutation operators are used to create new individuals in order to explore the solution space. The newly created individuals replace old individuals of the population at least partially, usually the worst ones, based on the fitness. The reproductive operators aim at producing variation in the population while maintaining 
the likelihood of converging to a global optimum. Population diversity enables the fruitful exploration of the search space. Without enough elitism, the search process becomes random and the algorithm cannot converge. Too much selection pressure increases the exploitation and the probability of turning the population homogeneous sooner, rather than later. Without enough diversity the search can be trapped prematurely in a local minimum, resulting in the algorithm failure. The efficiency of GAs is measured by their ability to find an appropriate balance between the exploration and exploitation of the search during the run.

Many algorithms coming from the specialized literature have successfully emerged, the most popular of which are based on sharing and crowing principles. They have been developed to counteract the convergence of the population to a single solution by maintaining a diverse population of individuals throughout the search. All the ideas developed are very promising and have given interesting results. However, the amount of simultaneous parameterization and the tuning of several parameters can often lead to extremely high computation costs without ensuring good results. Thus, more recently, several studies have emerged to counteract these shortcomings. They have focused on parameter adaptation (mutation, crossover rates and population sizes) and on the proposals of new variants (e.g. intelligent crossover, elitist recombination).

GAs have been applied to a number of design issues regarding both FIR and IIR filters. In [22], Lu and Tzeng used GAs to design finite-duration impulse response (FIR) digital filters with arbitrary log magnitude and phase responses. In [23], they extended their work to complex cases to design arbitrary complex finite-duration impulse response (FIR) digital filters. Evolutionary strategies have also been applied to the design and optimization of FIR filters for video signal processing applications, as presented in [24]. Recently, a hybrid genetic algorithm was proposed by Cen in [25]. The author designed the filters with coefficients restricted to the sum of signed powersof-two (SPoT) terms. The hybrid scheme was formed by integrating the main features of an adaptive genetic algorithm, simulated annealing and tabu search algorithms. Results showed that the normalized peak ripples of filters can be largely reduced. It should be noted that the efficiency of these algorithms is based the author's expertise in filter design and on the ability to design dedicated GAs. The usability of GAs for filter design can be improved by transferring more advances from the specialized GA literature.

\subsection{Particle Swarm Optimization}

The PSO method is a member of the broad category of Swarm Intelligence methods and is based on the collaboration between individuals often called particles. In a PSO algorithm, each particle is a candidate solution equivalent to a point in n-dimensional space. The algorithm is generally randomly initialized and the particles (candidate solutions) are placed randomly in the search space of the objective function [26]. The PSO successfully leads to a global optimum. The main concept of PSO is that the potential solutions are accelerated towards the best solutions. The particles iteratively evaluate the fitness of the candidate solutions and remember the location where they had their best fitness value. At each iteration, the particles move taking into account their best position but also the best position of their neighbors. The goal is to modify their trajectory so that they approach as close as possible to the optimum.

This optimum is achieved by an iterative procedure based on the processes of movement and intelligence in an evolutionary system. PSO focuses on cooperation rather than competition and there is no selection (at least in the basic version), the idea being that even a poor particle deserves to be preserved, perhaps because it is the one which ensures success in the future, precisely because it is unusual. It does not require gradient information of the objective function being considered, only its values. According to some results, PSO does not suffer from the problems encountered by other Evolutionary Computation techniques.

The basic procedure for implementing the PSO algorithm is as follows:

$V_{i}^{k+1}=\gamma * V_{i}^{k}+C_{1} * \operatorname{rand}_{1} *\left(\right.$ pbest $\left._{i}^{k}-X_{i}^{k}\right)+C_{2} * \operatorname{rand}_{2} *\left(\right.$ gbest $\left.^{k}-X_{i}^{k}\right)$

$X_{i}^{k+1}=X_{i}^{k}+V_{i}^{k}$

where $X_{i}^{k}$ and $V_{i}^{k}$ are respectively the position and velocity of the $\mathrm{i}^{\text {th }}$ particle at the $\mathrm{k}^{\text {th }}$ iteration, $p b e s t_{i}^{k}$ is the best position found so far by the particle and $g b e s t^{k}$ is the best of those found by all. 
$\gamma$ is the weighting function, $C_{1}$ and $C_{2}$ are positive weighting factors, rand $_{1}$ and rand $_{2}$ are random numbers between 0 and 1 . The basic procedure for implementing a PSO algorithm is given in Table 1 .

\begin{tabular}{ll}
\hline Table 1 & PSO algorithm (steps of PSO) \\
\hline
\end{tabular}

Step $1 \quad$ Initialize each particle vectors of the swarm by assigning a random velocity and position in the problem search space.

Step 2 Evaluate the fitness function values for each particle and compare the particle fitness value with the personal best solution $\left(\right.$ fitpbest $\left._{i}^{k-1}\right)$. If the current value is better than the $\left(\right.$ fitpbest $\left._{i}^{k-1}\right)$ value, update the value of $\left(\right.$ fitpbest $\left._{i}^{k}\right)$ and its best position $\left(\right.$ pbest $\left._{i}^{k}\right)$.

Step 3 Identify the particle that has the best fitness value. The value is its fitness function is identified as $\left(\right.$ fitgbest $\left.^{k}\right)$ and its position as $\left(\right.$ gbest $\left.^{k}\right)$.

Step $4 \quad$ Updating the velocities $\left(V_{i}^{k+1}\right)$ using the Eq.9 and the positions $\left(X_{i}^{k+1}\right)$ of all the particles using the Eq.10.

Step $5 \quad$ Replace the updated particle vectors as initial particle vectors for Step 2.

Step 6 Repeat steps 2-5 until the stopping criterion is met i.e. the maximum iteration cycles or the convergence to the good fitness value.

\subsection{Advanced PSO and filter design}

As pointed in Parrott 2006 [27], to effectively search such a space requires answering two further questions: how can particles update their known best positions in order to track optima and how can a population be arranged to balance the need to track existing optima against the need to distribute particles to search the remaining space for new optima? This question of balance between exploration and exploitation is at the heart of all evolutionary algorithms. The primary versions of PSO offer a novel strategy. It does not, however, manage the problems of fixing the algorithm's control parameters, premature convergence, stagnation and revisiting of the same solution over and again. Thus, several derived algorithms have been proposed in recent years to improve PSO efficiency [18]. Modifications to the original PSO essentially concern the parameter improvement and population structure. They have in common the goal of adding more flexibility, thus contributing to prevent the above problems [28, 29]. In this section, we focus on two PSO versions that are in line with the most recent advances in the evolutionary field. They have been especially developed for filter design, have proved their efficiency and have been advantageously compared to GA schemes.

NPSO [18] is an improved particle swarm optimization that proposes a new definition for the velocity vector and swarm updating. The key feature of the proposed modified inertia weight mechanism is to monitor the weights of particles. This inertia weight $\gamma$ plays the important role of balancing the global and local exploration abilities. A decreasing process for $\gamma$ is interesting as it encourages first space exploration and then exploitation. It cannot be a general solution especially when applied uniformly on all the particles. A slightly different approach further provides a well-balanced mechanism between global and local exploration abilities. It consists in monitoring the weights of particles individually by conditioning the decreasing process in order to prevent the particles from flying past the target position during the flight. The update of velocity is the same as (Eq. 9) but based on the following weighting function $\gamma_{q i}^{k}$ instead of $\gamma$ :

$$
\begin{aligned}
& \gamma_{q i}^{k}=\gamma_{\max }-\left(\gamma_{\max }-\gamma_{\min }\right) \frac{Z_{\text {iter }, q i}^{k}}{Z}, \text { if } V_{q i}^{k}\left(x_{i, g b e s t}^{k}-x_{q i}^{k}\right)>0 \\
& \gamma_{q i}^{k}=\gamma_{q i}^{k-1}, \text { if } V_{q i}^{k}\left(x_{i, g b e s t}^{k}-x_{q i}^{k}\right)<0
\end{aligned}
$$


where $q=1,2, \ldots, n_{p} ; i=1,2, \ldots, N . \gamma_{q i}^{k}$ is the element inertia weight $i$ of particle $q$ in iteration $k$.

$Z$ is a parameter controlling the linearly decreasing dynamic parameter framework descending from $\gamma_{\max }$ to $\gamma_{\min }$. According to the experimental results, the solution quality is improved and NPSO has the ability to converge to the best quality near optimal solution and possesses the best convergence characteristics in much shorter runtimes than other algorithms.

The craziness based PSO (CPSO) algorithm [19] is based on the unexpected behavior of a particle (bird or fish) in a flock that changes its direction suddenly and hence disturbs the organization. This is described by using a "craziness" factor and the core idea of CPSO is to model it by using a craziness variable. A craziness operator is introduced in the algorithm to ensure that the particle has a predefined craziness probability to maintain the diversity of the particles.

The velocity can be expressed:

$$
\begin{array}{r}
V_{i}^{k+1}=r_{2} * \operatorname{sign}\left(r_{3}\right) * V_{i}^{k}+\left(1-r_{2}\right) * C_{1} * r_{1} *\left(\text { pbest }_{i}^{k}-X_{i}^{k}\right) \\
+\left(1-r_{2}\right) * C_{2} *\left(1-r_{1}\right) *\left(\text { gbest }^{k}-X_{i}^{k}\right)
\end{array}
$$

$r_{1}, r_{2}$ and $r_{3}$ are random parameters $\in[0,1]$.

$\operatorname{sign}\left(r_{3}\right)$ is defined as:

$\operatorname{sign}\left(r_{3}\right)= \begin{cases}-1 & \text { if }\left(r_{3} \leq 0.05\right) \\ +1 & \text { if }\left(r_{3}>0.05\right)\end{cases}$

$\operatorname{rand}_{1}$ and rand $_{2}$ are independent random parameters. If both are large, both the personal and social experiences are over used and the particle is driven too far away from the local optimum. If both are small, both the personal and social experiences are not used fully and the convergence speed of the optimization technique is reduced. So, instead of taking independent rand ${ }_{1}$ and rand $_{2}$, one single random number $r_{1}$ is chosen so that when $r_{1}$ is large, (1$r_{1}$ ) is small and vice versa. Moreover, to control the balance of global and local searches, another random parameter $r_{2}$ is introduced.

$V_{i}^{k+1}=V_{i}^{k}+\operatorname{Pr}\left(r_{4}\right) * \operatorname{sign}\left(r_{4}\right) * V_{i}^{\text {craziness }}$

$r_{4}$ is a random parameter $\in[0,1]$.

$V_{i}^{\text {craziness }}$ is a random parameter $\in\left[V_{i}^{\text {craz_min }}, V_{i}^{\text {craz_max }}\right]$

$\operatorname{Pr}\left(r_{4}\right)$ and $\operatorname{sign}\left(r_{4}\right)$ are defined as:

$\operatorname{Pr}\left(r_{4}\right)= \begin{cases}1 & \text { if }\left(r_{4} \leq P_{\text {craz }}\right) \\ 0 & \text { if }\left(r_{4}>P_{\text {craz }}\right)\end{cases}$

$P_{\text {craz }}$ is a predefined craziness probability.

$\operatorname{sign}\left(r_{4}\right)= \begin{cases}+1 & \text { if }\left(r_{4} \geq 0.5\right) \\ -1 & \text { if }\left(r_{4}<0.5\right)\end{cases}$

\subsection{Our Adaptive Genetic Algorithm (AGA)}

One of the main problems for evolutionary algorithm designers is the fact that the performance of the algorithm is heavily dependent on design details, i.e. parameter values. Our AGA is devoted to FIR filter design and aims to be user-friendly for practitioners through its flexibility and capacity of self-tuning. It combines filter design knowhow and expertise in the application of various GAs for different optimization problems. A preliminary version of 
the algorithm has already been presented in Boudjelaba [30, 31]. This version has been improved by a better selection scheme and simplification of the parameter management.

From an initial calibration, the GA solves the design problem while repeatedly calibrating and re-calibrating itself by using several dedicated mechanisms. The initial calibration is necessary but not fundamental in terms of accuracy. It consists in generating a series of pre-calibrated parameters for the class of problems addressed by considering only the elementary operators of the GA. The goal is to reach GAs capable of regularly producing a genetic advance without oscillation and stagnation. Clearly, none of these static parameters are optimal but they are sufficient as starting points as it is possible to organize an adaptive strategy via dedicated mechanisms to avoid premature convergence and to accelerate convergence.

Thus, most of the parameters and operators are changed by the GA. The roles of the others are completely wellknown by the users.

Several complementary dedicated mechanisms assist GA convergence for a more efficient design and enable practical use by non-experts with different configurations (low pass, high pass, band pass and band cut). At any time, from the analysis of genetic evolution, the algorithm can shift by itself, changing its evolution at different scales if necessary through adaptation. The relevance of the GA is achieved through the strategy and not through the initial parameters, which are secondary.

The mechanisms are the following: firstly, genetic operators, and particularly the mutation operator, that are completely adapted to the level of the genetic process and therefore limit the simultaneous tuning of several static parameters; secondly, a ranking selection scheme that limits the promotion of extraordinary chromosomes, thus preventing premature convergence. To improve accuracy, the genetic exploration is driven by a fitness assignment strategy including the filter specificities. The selection process naturally promotes the best chromosomes. It integrates a function allowing the limitation of redundant chromosomes, hence promoting diversification. Our algorithm is therefore reinforced by a mechanism involving a refreshing process to reseed the population when necessary. We have developed a hybrid version of this algorithm that can slightly improve the results. The nonhybrid version is however sufficient to illustrate our argument here. The basic procedure for implementing an AGA is given in Table 2.

\begin{tabular}{ll}
\hline Table 2 & Adaptive Genetic Algorithm \\
\hline Step 1 & $\begin{array}{l}\text { Initialization: Define population size TP and denote a natural number } \mathrm{n} \text { as stop criterion; } \\
\text { code by real numbers; generate at random as initial population TP feasible individuals in } \\
\text { the problem search space. }\end{array}$
\end{tabular}

Step 2 Evaluate the fitness function values for each chromosome.

Step 3 Apply crossover operator.

Step 4 Apply Mutation operator with or without the diversification mechanism.

Step 5 Select chromosomes.

Step 6 Reseed or do not reseed the population by the refreshing process.

Step $7 \quad$ Updating the new population.

Step 8 Repeat steps 2-7 until the stopping criterion is met i.e. the maximum iteration cycles or the convergence to the good fitness value. 
Chromosome coding: the GA is a real-coded genetic algorithm (RCGA). A chromosome solution is directly represented by a vector of coefficients representing the filter itself.

The Crossover operator classically generates two children $p o p_{k}^{C h i l d}$ from two parents $i$ and $j$ according to the following rule:

$\left\{\begin{array}{l}X^{\text {Child } 1}=\left(X^{i}-X^{j}\right) * \lambda_{c 1}+X^{i} \\ X^{\text {Child } 2}=\left(X^{j}-X^{i}\right) * \lambda_{c 2}+X^{j}\end{array}\right.$

where $X^{i}\left(X^{j}\right)$ represents the individual $i(j), \lambda_{c 1}$ and $\lambda_{c 2}$ are random numbers between 0 and 1 . If Fitness(i)>Fitness (j) then $\lambda_{c 1}<\lambda_{c 2}$ in order to modify the best parent less. In our scheme the crossover operator produces $\mathrm{n}$ children that are treated via the selection scheme.

The Mutation operator is non-uniform, adaptive and directly applicable to each chromosome of the population individually. For the best chromosomes, the mutation rate is limited while for the worst chromosomes, it is encouraged. For a given chromosome, $P_{M}$ is applied to each gene.

$P_{M}=\left\{\begin{array}{cl}\frac{k_{1} *\left(f_{\max }-f\right)}{\left(f_{\max }-f_{\text {avg }}\right)} & f \geq f_{\text {avg }} \\ k_{2} & f<f_{\text {avg }}\end{array}\right.$

where $f_{\text {max }}$ and $f_{\text {avg }}$ are the maximum and average fitness values in the current population, respectively, and $f$ is the fitness value of the current chromosome. $k_{1}$ and $k_{2}$ are weighting parameters with $k_{1}$ and $k_{2} \leq 1$. The novelty lies in the conditions of use thereof, i.e., where the population evolves in a normal manner and is diverse. The simple idea consists in restarting a process of evolution that has stalled by refreshing more chromosomes. $P_{M}$ is increased once via the $k_{1}$ and $k_{2}$ values each time the population tends to get stuck in a local optimum (stagnation) and is decreased when the population is scattered in the search space. Thus, two couples of values, $\mathrm{k}_{1}$ and $\mathrm{k}_{2}$, are generated.

The level of mutation also takes feedback from the current state of the search. Each gene is modified accordingly. We suggest adopting a two-case version described as follows:

$X^{\text {new }}=\left\{\begin{array}{cc}X^{\text {old }} *\left(1+\lambda_{m 1}\right) & \text { if local minimum } \\ X^{\text {old }} *\left(1+\lambda_{m 2}\right) & \text { else }\end{array}\right.$

where $\lambda_{m 1}, \lambda_{m 2}$ are parameters depending on the genetic state $\left(\lambda_{m 1} \gg \lambda_{m 2}\right)$. The parameters $\mathrm{k}_{1}, \mathrm{k}_{2}, \lambda_{m 1}$ and $\lambda_{m 2}$ are pre-calibrated and fixed for the user. The mutation operator produces $n$ children like the crossover operation.

\section{Selection process}

The selection operator takes into account both elitism and diversity. The decision to select a given chromosome in the new population is based on two criteria; the first one is the fitness score. If there exists a chromosome in the candidate population (among the $4 * n$ ) with a higher score than the other candidates, it is likely to be selected. This is the elitist side of the process. However, if a candidate fulfills this elitist criterion but presents a comparable structure with a chromosome that has already been selected, it is likely to be rejected. This is the diversity side of the process. Our scheme is iterative and implicitly includes the well-known crowding and niching concepts [31]. The computational cost is optimized. The process requires the definition of similarity between two chromosomes. The distance is calculated only if two chromosomes are similar from the fitness space. For a class of filters some coefficients are more relevant and can be selected. A reduced space of 2 dimensions has been determined via a statistical procedure. Thus, similarity is expressed via a threshold that has been calibrated.

The algorithm starts with the best chromosome called current. This chromosome is automatically selected and first compared to the second called inspected via the fitness function. If there is no fit between the two, the second becomes current and the procedure continues. Otherwise, the comparison is done via a gene metric to avoid discarding two chromosomes having similar fitness values while being genetically different. Inspected is discarded 
if there is no fit between them in this space. In this case the first chromosome is still current. The comparison is then done with the third chromosome which becomes inspected. The procedure continues until the whole population has been inspected. If less than $n$ chromosomes $\left(n_{s}\right)$ have been selected, $\left(n-n_{s}\right)$ are randomly retrieved among the non- selected ones $\left(4 n-n_{s}\right)$. Chromosomes, even with a relatively low fitness, can survive via this selection process that increases the chances of improving performances via genetic operators.

\section{Detection of stagnation (local minimum) and breaking process}

The process of verification of the presence of a local minimum can be summarized in this equation:

$\left\{\begin{array}{l}\text { If }\left(f_{\text {avg }} \leq t \mathrm{~h}_{1} \text { and } \Delta_{\text {fitness }(\text { avg })} \leq t \mathrm{~h}_{2} \text { and } \frac{f_{\text {max }}-f_{\text {avg }}}{f_{\text {avg }}} \leq t h_{3}\right) \text { Presence of a local minimum } \\ \text { Else No presence of a local minimum }\end{array}\right.$

The detection of a local minimum is based only on the fitness values. It presents a very low computational cost as it is based on simple calculations.

According to a given metric, one way of detecting it aims at calculating or estimating the percentage of similar chromosomes within the population and reseeding the population accordingly. It is possible to work directly on the fitness space by comparing the best chromosome and the average fitness function. In both cases, breaking is subject to the level of the genetic advance.

$\left\{\begin{array}{l}\text { if } \Delta_{\text {fitness }}\left(f\left(\text { ch }_{\text {best }}\right), f\left(\text { ch }_{\text {ave }}\right)\right) \leq \Delta f_{\text {min }} \quad \text { and } \\ f\left(\mathrm{ch}_{\text {best }}\right) \leq f_{\text {end }} \text { breaking process active } \\ \text { breaking process inactive }\end{array}\right.$

where $\Delta f_{\min }$ and $f_{\text {end }}$ are the different thresholds and $f(x)$ the fitness of chromosome $x$.

An elementary calibration procedure makes it possible to find confidence intervals for the associated parameters. The user can play with the confidence intervals to inject more or less dynamics to the process.

\section{Experimental results}

The particle in the PSO and the chromosome in the GA are composed of the set of filter coefficients with respect to the impulse response sequence and many particles (chromosomes) further a population.

\section{Parameter setting}

\section{Basic genetic parameters}

These parameters are pre-calibrated and do not need to be tuned.

- The initial population size Size $_{\mathrm{pop}}$ is 120 . The evolutionary cycle is repeated until 500 generations.

- The values of parameters such as crossover and mutation probability determine $C_{R}, P_{M}$, and $k_{1}, k_{2}$, $\lambda_{m 1}, \lambda_{m 2}$, to a greater extent, whether an algorithm will find an optimal solution with speed, accuracy and diversity. The table 3 shows the performance obtained for different values of the parameters mentioned above. 
Table 3 Parameter setting of the AGA

\begin{tabular}{|c|c|c|c|c|c|}
\hline & Parameters & $\begin{array}{l}\text { Max stop } \\
\text { band } \\
\text { ripple }\end{array}$ & $\begin{array}{l}\text { Max pass } \\
\text { band } \\
\text { ripple }\end{array}$ & $\begin{array}{l}\text { Average } \\
\text { error }\end{array}$ & Ite \\
\hline \multirow{13}{*}{ 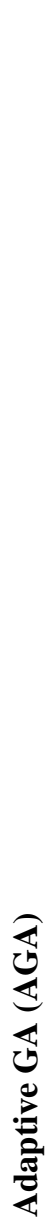 } & $\begin{array}{c}C_{R}=100 \%, P_{M}=40 \%, k_{1}=0.1 \%, k_{2}=1 \% \\
\lambda_{m 1}=2 * \text { rand }, \lambda_{m 2}=0.2 * \text { rand }\end{array}$ & 0.0119 & 0.0831 & 0.0458 & 215 \\
\hline & $\begin{array}{c}C_{R}=80 \%, P_{M}=40 \%, k_{1}=0.1 \%, k_{2}=1 \% \\
\lambda_{m 1}=2 * \text { rand }, \lambda_{m 2}=0.2 * \text { rand }\end{array}$ & 0.0142 & 0.0906 & 0.0492 & 308 \\
\hline & $\begin{array}{c}C_{R}=40 \%, P_{M}=40 \%, k_{1}=0.1 \%, k_{2}=1 \% \\
\lambda_{m 1}=2 * \text { rand }, \lambda_{m 2}=0.2 * \text { rand }\end{array}$ & 0.0145 & 0.0918 & 0.0509 & 423 \\
\hline & $\begin{array}{c}C_{R}=100 \%, P_{M}=1 \%, k_{1}=0.1 \%, k_{2}=1 \% \\
\lambda_{m 1}=2 * \text { rand }, \lambda_{m 2}=0.2 * \text { rand }\end{array}$ & 0.0128 & 0.0865 & 0.0471 & 377 \\
\hline & $\begin{array}{c}C_{R}=100 \%, P_{M}=80 \%, k_{1}=0.1 \%, k_{2}=1 \% \\
\lambda_{m 1}=2 * \text { rand }, \lambda_{m 2}=0.2 * \text { rand }\end{array}$ & 0.0124 & 0.0852 & 0.0464 & 223 \\
\hline & $\begin{array}{c}C_{R}=100 \%, P_{M}=40 \%, k_{1}=0.01 \%, k_{2}=1 \% \\
\lambda_{m 1}=2 * \text { rand }, \lambda_{m 2}=0.2 * \text { rand }\end{array}$ & 0.0122 & 0.0847 & 0.0469 & 358 \\
\hline & $\begin{array}{c}C_{R}=100 \%, P_{M}=40 \%, k_{1}=0.9 \%, k_{2}=1 \% \\
\lambda_{m 1}=2 * \text { rand }, \lambda_{m 2}=0.2 * \text { rand }\end{array}$ & 0.0120 & 0.0835 & 0.0463 & 310 \\
\hline & $\begin{array}{c}C_{R}=100 \%, P_{M}=40 \%, k_{1}=0.1 \%, k_{2}=10 \% \\
\lambda_{m 1}=2 * \text { rand }, \lambda_{m 2}=0.2 * \text { rand }\end{array}$ & 0.0198 & 0.1012 & 0.0470 & 302 \\
\hline & $\begin{array}{c}C_{R}=100 \%, P_{M}=40 \%, k_{1}=0.1 \%, k_{2}=0.2 \% \\
\lambda_{m 1}=2 * \text { rand }, \lambda_{m 2}=0.2 * \text { rand }\end{array}$ & 0.0123 & 0.0834 & 0.0468 & 359 \\
\hline & $\begin{array}{c}C_{R}=100 \%, P_{M}=40 \%, k_{1}=0.1 \%, k_{2}=1 \% \\
\lambda_{m 1}=5 * \text { rand }, \lambda_{m 2}=0.2 * \text { rand }\end{array}$ & 0.0163 & 0.0985 & 0.0466 & 389 \\
\hline & $\begin{array}{c}C_{R}=100 \%, P_{M}=40 \%, k_{1}=0.1 \%, k_{2}=1 \% \\
\lambda_{m 1}=0.5 * \text { rand }, \lambda_{m 2}=0.2 * \text { rand }\end{array}$ & 0.0151 & 0.0936 & 0.0502 & 374 \\
\hline & $\begin{array}{c}C_{R}=100 \%, P_{M}=40 \%, k_{1}=0.1 \%, k_{2}=1 \% \\
\lambda_{m 1}=2 * \text { rand }, \lambda_{m 2}=2 * \text { rand }\end{array}$ & 0.0172 & 0.0989 & 0.0518 & 415 \\
\hline & $\begin{array}{c}C_{R}=100 \%, P_{M}=40 \%, \quad k_{1}=0.1 \%, k_{2}=1 \% \\
\lambda_{m 1}=2 * \text { rand }, \lambda_{m 2}=0.05 * \text { rand }\end{array}$ & 0.0164 & 0.0974 & 0.0501 & 428 \\
\hline
\end{tabular}

The parameters of AGA were determined from several tests. The values of these parameters can give algorithms with optimal performances, i.e. which reduce errors and minimize the computation time required for convergence.

In what follows (table 4), we list the parameters and their values used in the tests.

Table 4 Parameters of AGA

\begin{tabular}{|c|c|c|}
\hline & Parameters & \\
\hline $\begin{array}{l}\text { Adaptive GA } \\
\text { (AGA) }\end{array}$ & 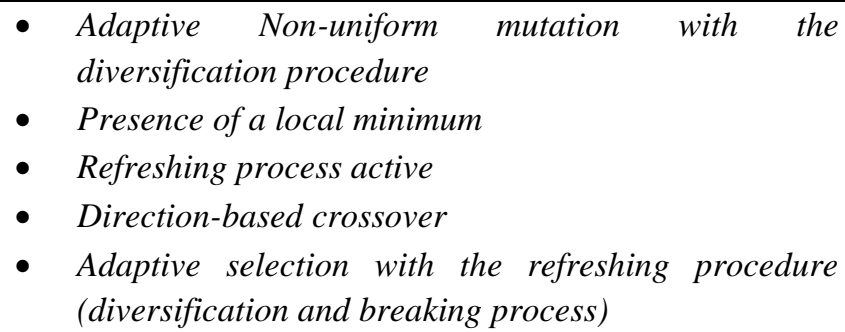 & $\begin{array}{l}k_{1}=0.1 \%, k_{2}=1 \% \\
k_{1}=10 \%, k_{2}=30 \% \\
P_{M}=40 \% \\
C_{R}=100 \%\end{array}$ \\
\hline
\end{tabular}




\section{Domain parameters}

- $\quad$ The value of $W$ which gives the best results is 1 in the pass band and (5/8) ${ }^{2}$ in the stop band. These values were calibrated after several tests for different values of $W$ and filter configurations.

Table 5 summarizes the best chosen parameters for all the algorithms (GA, AGA, PSO and NPSO, CPSO) used for the design of FIR filters. In this paper, there is no comparison with classical approaches of filter design as many studies dealing with evolutionary approaches have already dealt with this and lead to similar conclusions. For the same reason, the most basic GA with static parameters and classic genetic operators for crossover, mutation and selection has not been included in the comparison. Although the results obtained with this type of filter are interesting, they cannot be extrapolated for other problems as they depend on human ability to tune parameters. The proper selection of parameters plays an important role in the convergence profile of the respective algorithms. They were selected from the reference papers for PSO algorithms [18, 19]. The results presented for each algorithm are the best (according to the average error) among 5 successive runs launched with the same parameters. The repeatability of the results is quite good, which underlines the consistency of the algorithms: the ratio between the worst and the best error is less than $5 \%$ for all the algorithms except PSO which generally presents a larger deviation, though less than $10 \%$.

These algorithms are also used for the design of high pass FIR filters and have got the same performance profiles as low pass FIR filters.

Table 5 Parameters of each algorithm

\begin{tabular}{|l|l|l|l|l|l|}
\hline Parameters & SGA & AGA & PSO & NPSO & CPSO \\
\hline $\begin{array}{l}\text { Frequency sampling } \\
\text { number }\end{array}$ & 128 & 128 & 128 & & \\
\hline Population size & 120 & 120 & 120 & 120 & 120 \\
\hline $\begin{array}{l}\text { Number max of } \\
\text { iterations }\end{array}$ & 500 & 500 & 500 & 500 & 500 \\
\hline Crossover & $\begin{array}{l}\text { direction-based } \\
\text { crossover }(1 \\
\text { child) }\end{array}$ & $\begin{array}{l}\text { direction-based crossover } \\
(2 \text { children })\end{array}$ & - & - & - \\
\hline Crossover rate & $100 \%$ & $100 \%$ & Adaptive non-uniform & - & - \\
\hline Mutation & $\begin{array}{l}\text { Non-uniform } \\
\text { mutation }\end{array}$ & diversification procedure & - & - & - \\
\hline Mutation rate & $0.5 \%$ & Adaptive mutation rate & - & - & - \\
\hline Refreshing process & - & Active & - & - & - \\
\hline$C_{1}$ & - & - & 2.05 & 2.05 & 2.05 \\
\hline$C_{2}$ & - & - & 2.05 & 2.05 & 2.05 \\
\hline$V_{i}^{\text {min }}$ & - & - & 0.01 & 0.01 & 0.01 \\
\hline$V_{i}^{\text {max }}$ & - & - & 1 & 1 & 1 \\
\hline$\gamma^{\text {min }}$ & - & - & 0.4 & 0.4 & - \\
\hline$\gamma^{\text {max }}$ & - & - & 1 & 1 & - \\
\hline$P_{c r}$ & - & - & - & 0.3 \\
\hline$v_{\text {craziness }}$ & - & - & - & 0.001 \\
\hline
\end{tabular}

The tests in $[15,17,18]$ deal with FIR low-pass filters of order 20 and 30 . To this end, the simulation was performed to design the low pass FIR filter with filter coefficient lengths of 21, 31 and 41 . The frequency sampling number chosen was 128 . 


\section{Example 1}

$$
D(\omega)=\left\{\begin{array}{lc}
1 & 0 \leq \omega \leq 0.5 \\
0 & \omega>0.5
\end{array}\right.
$$

Fig. 1 and 2 show the magnitude response for the low-pass FIR filter of order 40 obtained using GA, AGA, PSO, NPSO and CPSO algorithms and the convergence profiles of each algorithm, respectively.

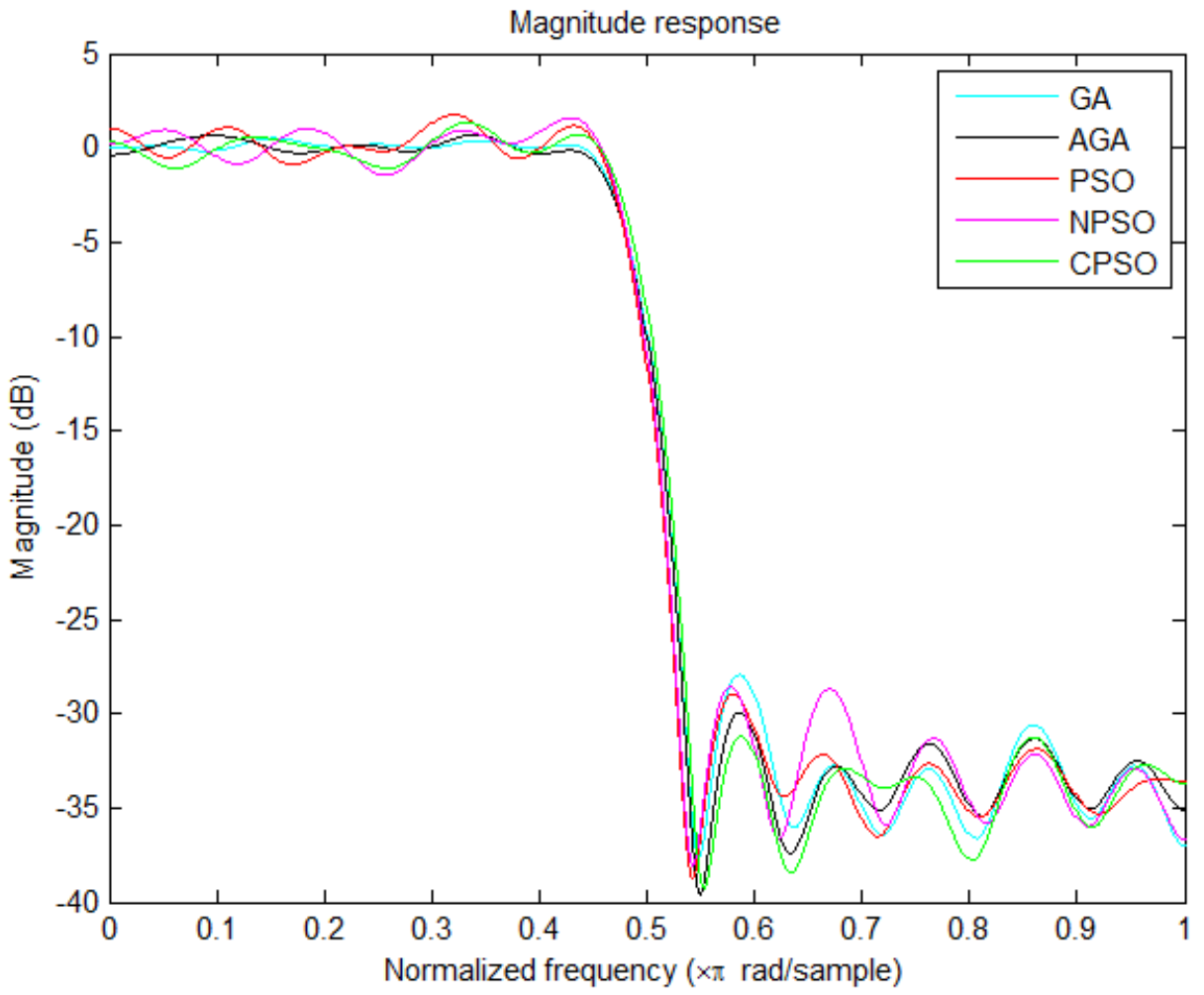

Fig. 1 Magnitude response $(d B)$ of the $40^{\text {th }}$ order low-pass FIR filters $\left(\omega_{c}=0.5\right)$ designed using GA, AGA, PSO, NPSO and CPSO 


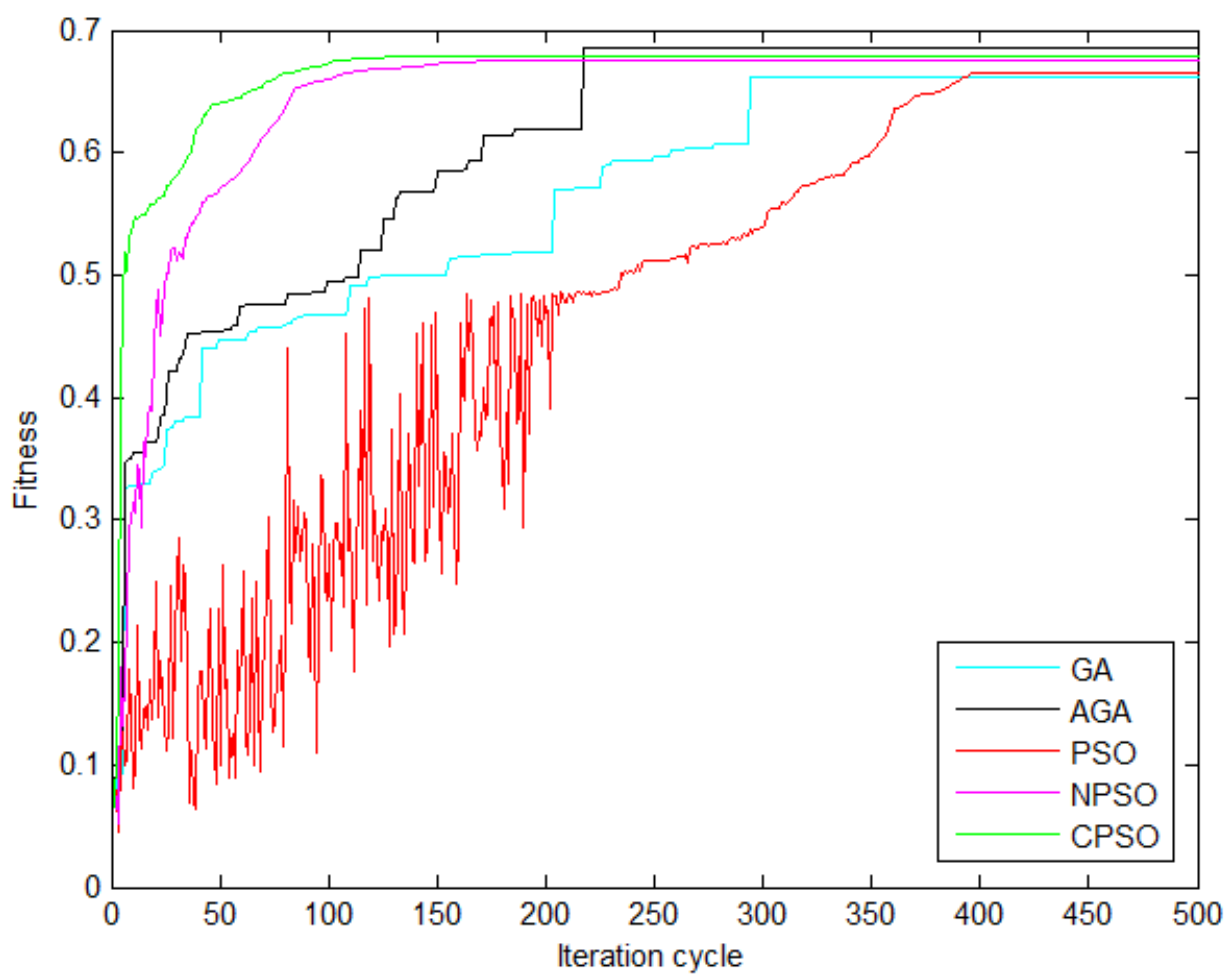

Fig. 2 Convergence profile for GA, AGA, PSO, NPSO and CPSO in case of $40^{\text {th }}$ order Low Pass FIR filters $\left(\omega_{c}=0.5\right)$

Fig. 2 shows the complete comparison of the evolution of the fitness function given by the different algorithms. This curve evidences the benefits of CPSO in terms of the number of iterations needed for convergence. Compared with the other algorithms, it is clear that when using sophisticated versions of PSO algorithms, all the particles tend to converge to the best solution quickly in most cases, whereas the basic version of PSO produces the worst result and presents many oscillations at the start of the process.

Table 6 summarizes the comparative results of different performance parameters obtained using GA, AGA, PSO, NPSO and CPSO algorithms for a low-pass FIR filter of order 40.

Table 6 Performances of each algorithm $\left(2 * N+1=41, \omega_{c}=0.5\right)$

\begin{tabular}{|l|l|l|l|l|l|}
\hline Parameters & SGA & AGA & PSO & NPSO & CPSO \\
\hline $\begin{array}{l}\text { Maximum stop band } \\
\text { ripple }\end{array}$ & 0.0201 & 0.0119 & 0.0155 & 0.0173 & 0.0087 \\
\hline $\begin{array}{l}\text { Maximum pass band } \\
\text { ripple }\end{array}$ & 0.0604 & 0.0831 & 0.2252 & 0.2015 & 0.1648 \\
\hline Average error & 0.0512 & 0.0458 & 0.0644 & 0.0641 & 0.0509 \\
\hline Numbers of iterations & 294 & 215 & 398 & 172 & 128 \\
\hline
\end{tabular}

The GA and AGA algorithms give the best value of the maximum pass band ripple but the AGA algorithm gives the best average error. The NPSO and CPSO converge to the desired value after 172 and 128 iterations respectively. These results indicate that the AGA and PSO give better results in terms of error and number of iterations. This simulation result clearly demonstrates the superiority of the Adaptive GA and craziness PSO over the other methods used in this paper (GA, PSO, NPSO). 
Example 2

$$
D(\omega)=\left\{\begin{array}{lc}
1 & 0 \leq \omega \leq 0.5 \\
0 & \omega>0.5
\end{array}\right.
$$

Fig. 3 shows the magnitude response obtained for the low-pass FIR filter of order 20 using the GA, AGA, PSO, NPSO and CPSO algorithms. Table 7 shows the maximum stop band ripple, average error and number of iterations obtained when all the algorithms are employed for the design of a low pass FIR filter of order 20. The algorithms can be compared in terms of their convergence speeds. Fig. 4 shows the plot of maximum fitness values against the number of iterations when all the algorithms cited in this paper are applied. From Fig. 4, it can be seen that the CPSO is significantly faster than the other algorithms at finding the optimum filter. The AGA converges to a much better fitness in a reasonable number of iterations. Table 7 supports our claim that the AGA is more efficient for the design of FIR filters. Except for PSO, all the algorithms present a similar curve at the start but the level of fitness is continuously worse for GA. Concerning PSO, oscillations are always present in the fitness curve but the fitness reached at the convergence is better than that of GA and the same as that of NPSO. It is interesting to compare GA and AGA: one can observe that the fitness curves change step by step. This differs from the PSO versions which present a more continuous progression. The GA versions have non-uniform mutation in common; the difference between them lies in the level of the steps. This can be interpreted as a better capacity for AGA of finding better regions of the search space. This dynamic is to a certain extent dissociated from the genetic advance which can leave practitioners without any help for a given situation.

Table 7 Performances of each algorithm $\left(2 * N+1=21, \omega_{c}=0.5\right)$

\begin{tabular}{|l|l|l|l|l|l|}
\hline Parameters & SGA & AGA & PSO & NPSO & CPSO \\
\hline $\begin{array}{l}\text { Maximum stop band } \\
\text { ripple }\end{array}$ & 0.0088 & 0.0191 & 0.0076 & 0.0097 & 0.0086 \\
\hline $\begin{array}{l}\text { Maximum pass band } \\
\text { ripple }\end{array}$ & 0.1989 & 0.1697 & 0.2392 & 0.2342 & 0.2468 \\
\hline Average error & 0.0625 & 0.0598 & 0.0755 & 0.0799 & 0.0802 \\
\hline Numbers of iterations & 302 & 177 & 419 & 99 & 118 \\
\hline
\end{tabular}

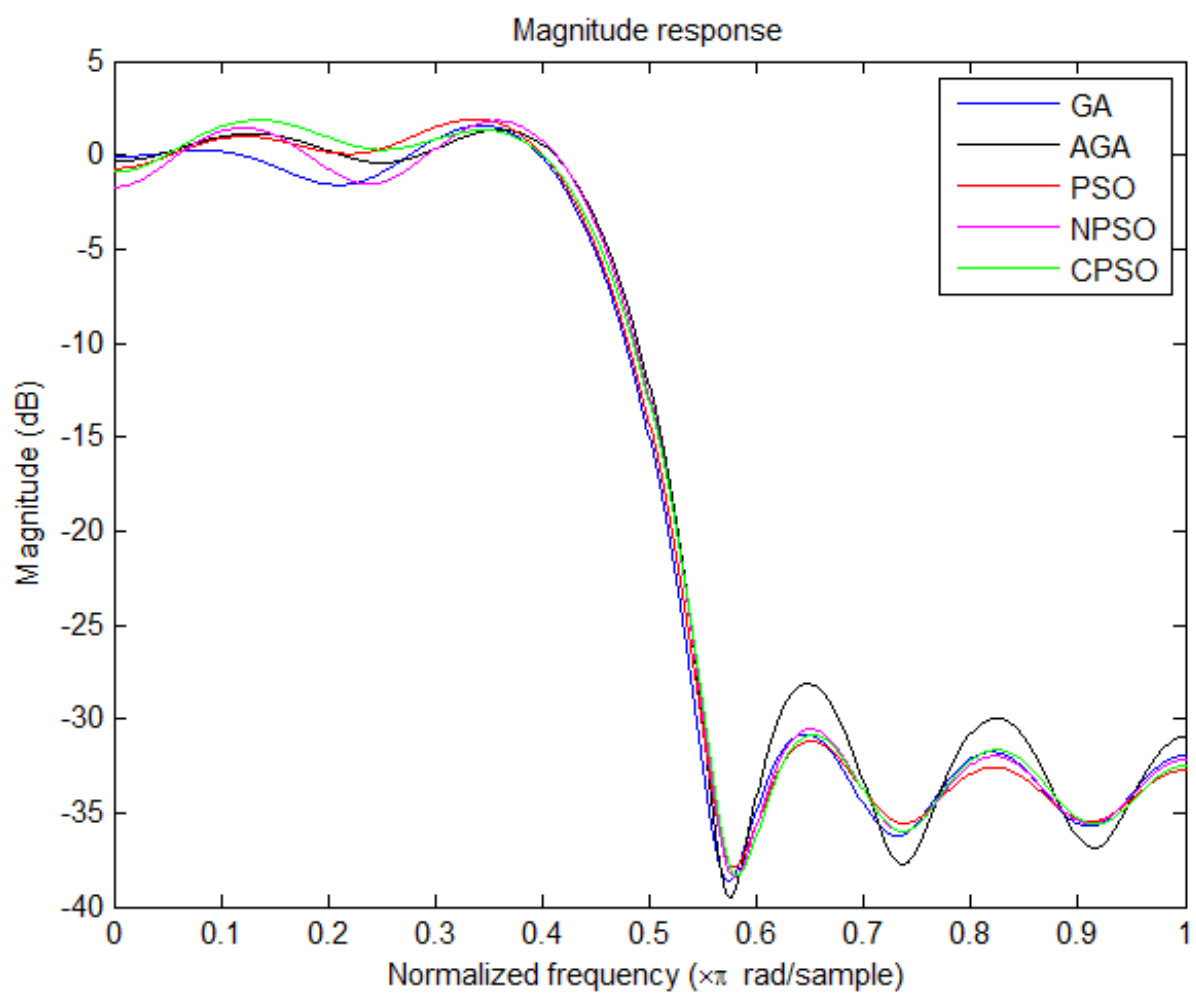

Fig. 3 Magnitude response $(\mathrm{dB})$ of the $20^{\text {th }}$ order low-pass FIR filters $\left(\omega_{c}=0.5\right)$ designed using GA, AGA, PSO, NPSO and CPSO 


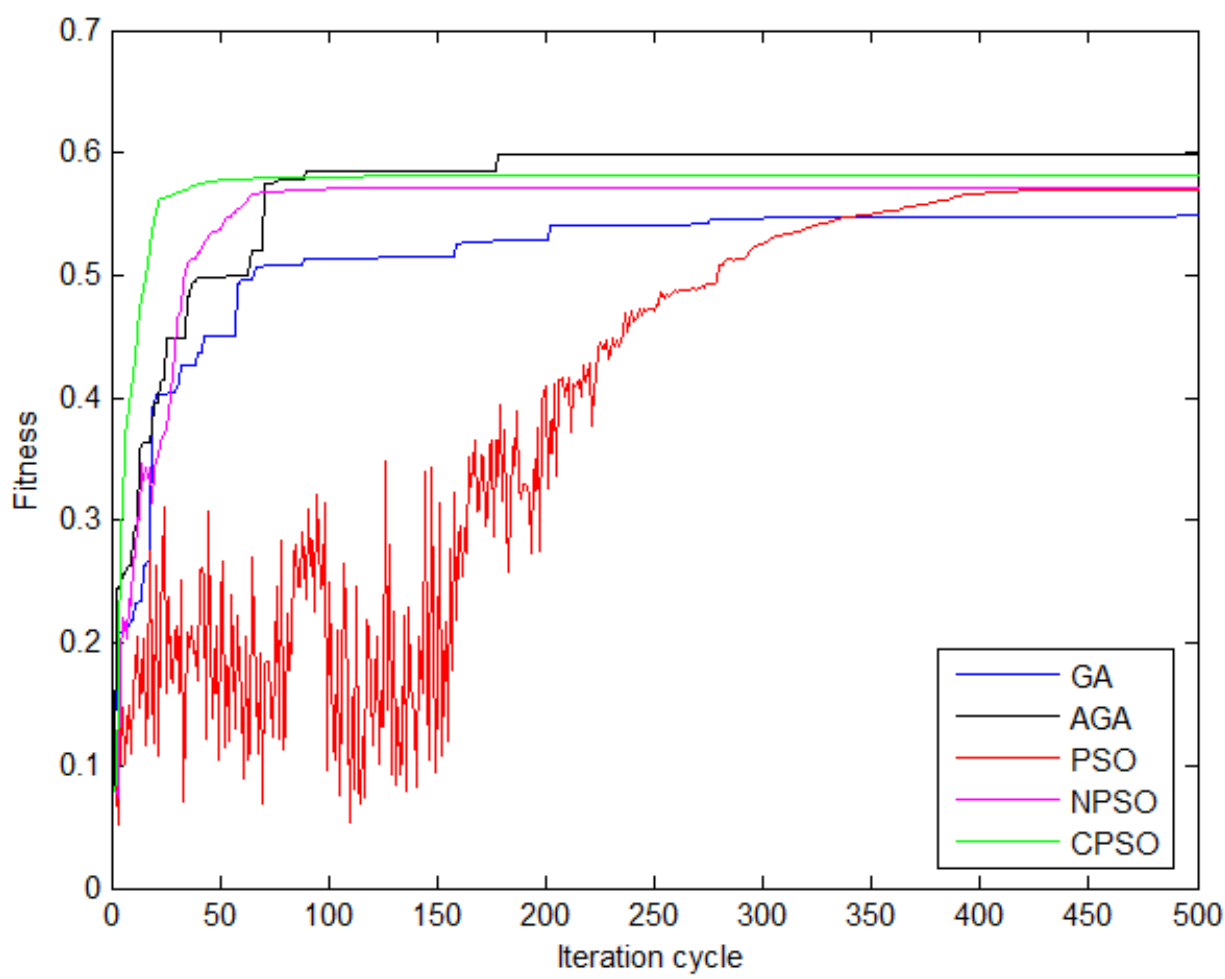

Fig. 4 Convergence profile for GA, AGA, PSO, NPSO and CPSO in case of $20^{\text {th }}$ order Low Pass FIR filters $\left(\omega_{c}=0.5\right)$

Example 3

$$
D(\omega)=\left\{\begin{array}{lc}
1 & 0 \leq \omega \leq 0.4 \\
0 & \omega>0.4
\end{array}\right.
$$

Table 8 gives the complete comparison between the GA, AGA, PSO, NPSO and CPSO algorithms for the design of an FIR filter of order 40. Fig. 5 shows the magnitude response for the low-pass FIR filter of order 40 obtained using the GA, AGA, PSO, NPSO and CPSO algorithms.

Table 8 Performances of each algorithm $\left(2 * N+1=41, \omega_{c}=0.4\right)$

\begin{tabular}{|l|l|l|l|l|l|}
\hline Parameters & SGA & AGA & PSO & NPSO & CPSO \\
\hline $\begin{array}{l}\text { Maximum stop band } \\
\text { ripple }\end{array}$ & 0.0313 & 0.0361 & 0.0112 & 0.0108 & 0.0100 \\
\hline $\begin{array}{l}\text { Maximum pass band } \\
\text { ripple }\end{array}$ & 0.0939 & 0.0507 & 0.1971 & 0.1888 & 0.1320 \\
\hline Average error & 0.0763 & 0.0679 & 0.0794 & 0.0735 & 0.0717 \\
\hline Numbers of iterations & 365 & 215 & 487 & 168 & 134 \\
\hline
\end{tabular}




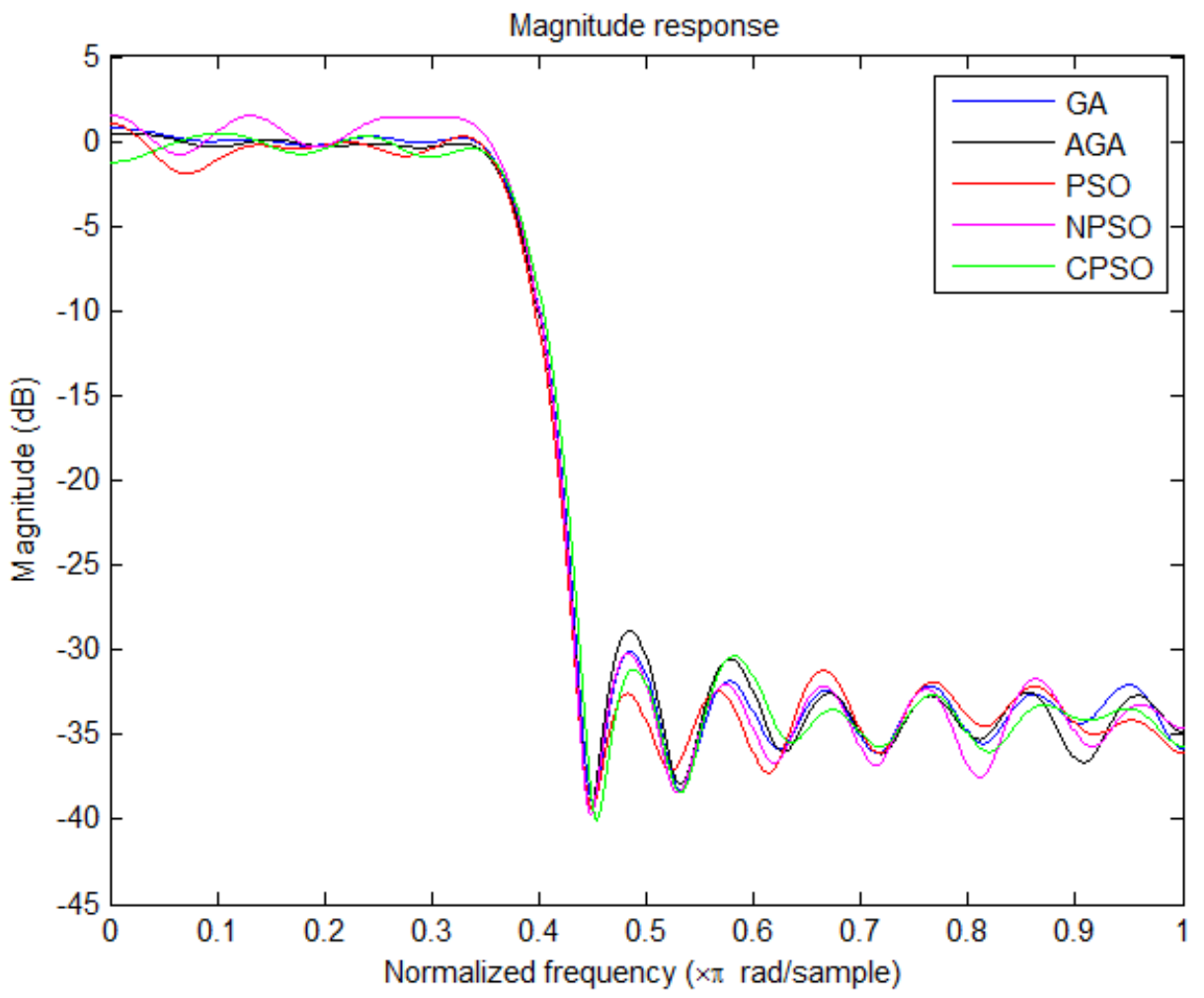

Fig. 5 Magnitude response $(\mathrm{dB})$ of the $40^{\text {th }}$ order low-pass FIR filters $\left(\omega_{c}=0.4\right)$ designed using GA, AGA, PSO, NPSO and CPSO

Table 8 shows that the best maximum pass band ripple achieved for a low pass filter using the AGA is 0.0507 . It is observed from Table 8 that CPSO achieves the best iteration cycle for convergence. Only 134 iterations are required while 487 are necessary for PSO for a poorer efficiency. For this example, the NPSO and CPSO algorithms present similar performances. AGA has a better magnitude response, pass band ripple and average error than the other algorithms. 215 iterations are however necessary to reach convergence.

\section{Example 4}

$$
D(\omega)=\left\{\begin{array}{lc}
1 & 0 \leq \omega \leq 0.6 \\
0 & \omega>0.6
\end{array}\right.
$$

As seen from Fig. 6, for the pass band region, the AGA, NPSO and CPSO produce a better response than that of GA and PSO. For the stop band region, the filter designed by PSO produces a better response than the others. For this example, the average error obtained with AGA $(0.0185)$ is much better than the ones obtained by the other algorithms (from 0.0419 to 0.0674 ). Table 9 summarizes the performances of each algorithm. 


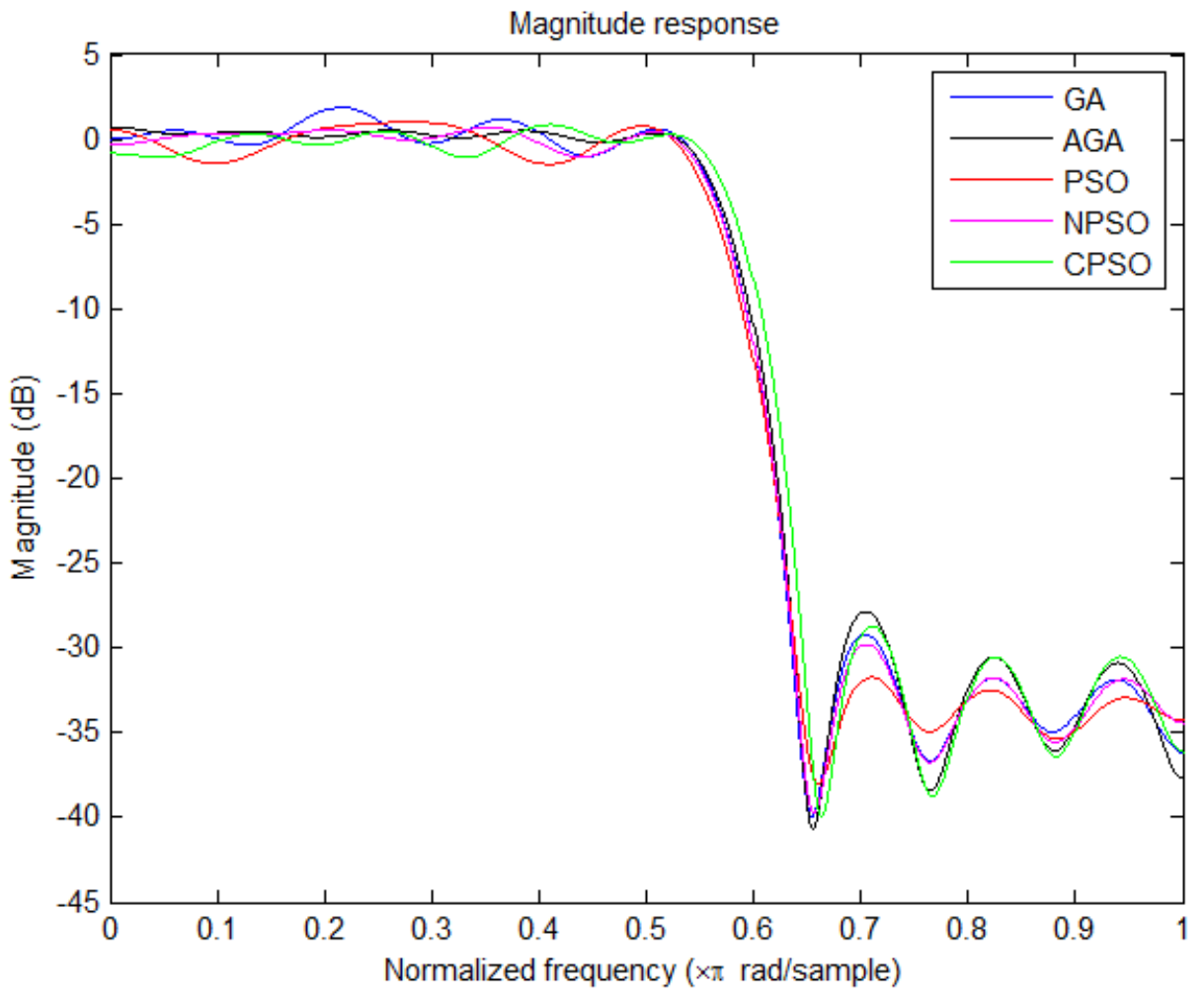

Fig. 6 Magnitude response $(\mathrm{dB})$ of the $30^{\text {th }}$ order low-pass FIR filters $\left(\omega_{c}=0.6\right)$ designed using GA, AGA, PSO, NPSO and CPSO

Table 9 Performances of each algorithm $\left(2 * N+1=31, \omega_{c}=0.6\right)$

\begin{tabular}{|l|l|l|l|l|l|}
\hline Parameters & SGA & AGA & PSO & NPSO & CPSO \\
\hline $\begin{array}{l}\text { Maximum stop band } \\
\text { ripple }\end{array}$ & 0.0145 & 0.0194 & 0.0072 & 0.0124 & 0.0165 \\
\hline $\begin{array}{l}\text { Maximum pass band } \\
\text { ripple }\end{array}$ & 0.2302 & 0.0191 & 0.1551 & 0.1129 & 0.1144 \\
\hline Average error & 0.0674 & 0.0185 & 0.0665 & 0.0419 & 0.0422 \\
\hline Numbers of iterations & 297 & 203 & 471 & 135 & 163 \\
\hline
\end{tabular}

\section{Usability studies}

In order to evaluate the usability (repeatability, performance and number of iterations) of AGA, we designed the filter in example 1, by running the algorithm 10 and 100 times in succession. Results in terms of maximum ripple in stop band, maximum ripple in pass band and average error are given in Fig. 7 and 8. 

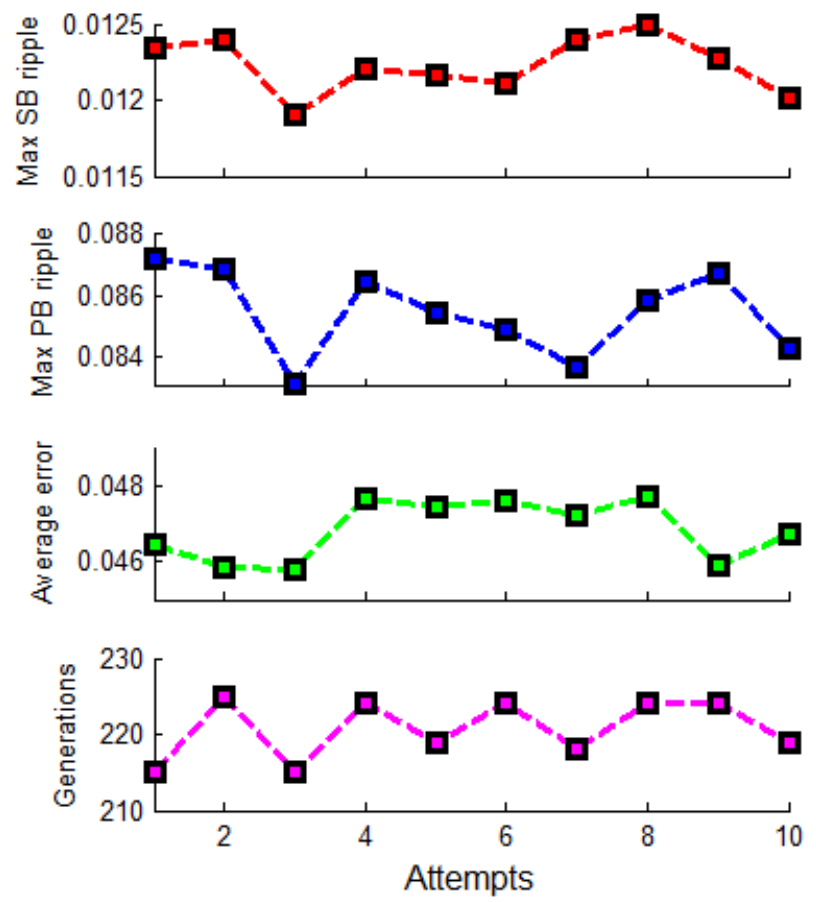

Fig. 7 Performances obtained for 10 runs (Variation and evolution of performance for 10 executions).
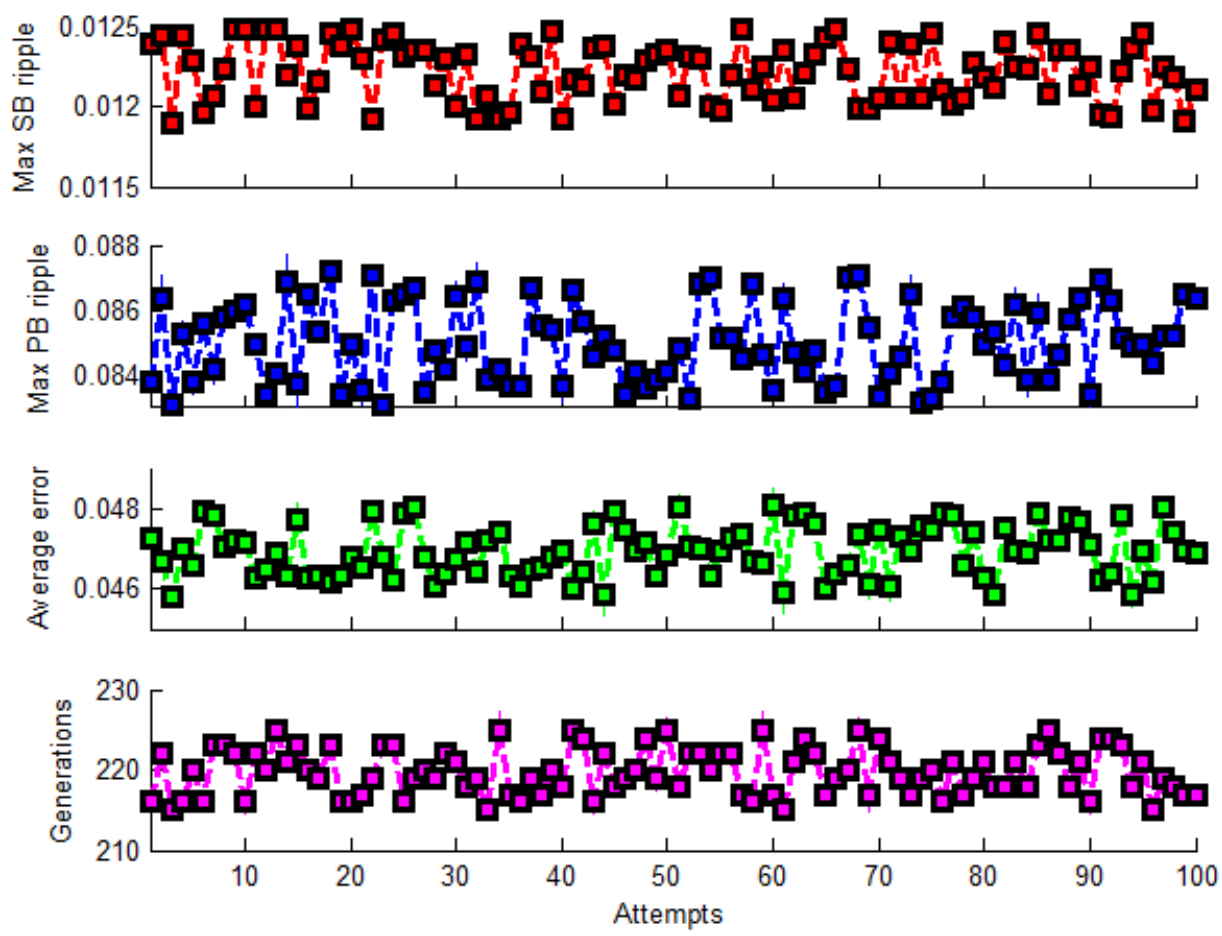

Fig. 8 Performances obtained for 100 runs (Variation and evolution of performance for 100 executions).

To investigate further, we generated 100 filters (example 1) and launched the runs. Table 10 represents the average error and peak errors obtained. The error variations (mean and peak) on large scales of tests are very small, demonstrating the consistency of our algorithm. Some cases are more distinguishable when the peak error is 
analyzed but the amount of variation remains very small. And for each run, the algorithm gives satisfactory results in terms of performance.

Table 10 Mean and standard deviation obtained for 100 runs

\begin{tabular}{|c|c|c|c|}
\cline { 2 - 4 } \multicolumn{1}{c|}{} & Parameters & Mean & Standard deviation \\
\hline \multirow{3}{*}{$\begin{array}{c}\text { Adaptive GA } \\
\text { (AGA) }\end{array}$} & Maximum stop band ripple & 0.0122 & $1.7962 \times 10^{-4}$ \\
\cline { 2 - 4 } & Maximum pass band ripple & 0.0851 & $12 \times 10^{-4}$ \\
\cline { 2 - 4 } & Average error & 0.0469 & $6.5761 \times 10^{-4}$ \\
\hline
\end{tabular}

\section{Discussion and perspectives}

The merits of PSO lie in its simplicity of implementation as well as the fact that its convergence can be controlled via few parameters. However, the basic version, despite its interest, produces worse and less repeatable results than the other, more sophisticated, algorithms. There are control parameters involved in PSO, and appropriate setting of these parameters remains a key point for success. Some form of trial-and-error tuning is necessary for each particular instance of a filter design problem. Although PSO is probably simpler than GA, the situation for practitioners is similar. No conclusion can be drawn concerning the superiority of one method over the other in terms of performances. It should be said that basic versions of GAs are less user-friendly and are more dependent on the efforts and ability to manage parameter tuning than the algorithms themselves.

NPSO and particularly CPSO are more sophisticated. They clearly produce better results than the basic version of PSO. While control parameters are involved in the algorithms, they involve some mechanisms that can optimize the search and achieve rather good results. They make it possible to update the swarm's knowledge of the environment and the balance between exploration and exploitation is better managed. NPSO and CPSO rely on the same general idea: at the beginning they facilitate global search, but focus more on space exploitation at the end. Setting appropriate parameters for a class of problems does not appear to be difficult. CPSO embeds some intelligence making it more self-tuning, which gives a significant difference in the results.

As can be noted from this study, the NPSO and CPSO iteration cycles are better than those of the AGA, GA and PSO. AGA, NPSO and CPSO converge to their respective minimum ripple magnitude in less than 250 iterations. Furthermore, NPSO and CPSO yield suboptimal higher values of error but AGA yields near optimal (least) error values consistently in both cases. Note that 100 iterations for PSO correspond to $3.44 \mathrm{~s}$ and $19.28 \mathrm{~s}$ for AGA, thus amplifying the difference in the convergence rate between PSO and AGA. We consider that this is a major strength of these algorithms. In view of the above fact, it may finally be inferred that the performance of the AGA technique is better than that of NPSO and CPSO but needs more time to explore and exploit the search space. A plausible explanation for this superiority is that in a classic GA scheme, it is difficult in practice for a chromosome to occupy any state of the search as multiple iterations are required. Then, when approaching convergence, the necessary selectivity introduced in the algorithm penalizes diversity. In our process, diversity is managed better through the different mechanisms that enable other zones of the search space to be explored even when convergence is almost reached and that increase the probability of improving the general performances. This can be easily observed by analyzing the shape of the best fitness functions. Unlike the other algorithms that present a strong continuity, the best fitness function for AGA moves with a given continuity but via consecutive steps.

Classic GAs are reputed to be efficient at exploring the entire search space but poor at finding an accurate local optimum. For this reason, they are often combined with local approaches to compensate for this weakness. PSO schemes explore differently. Our simulations prove that at the starting they have a faster convergence rate but rapidly become inefficient at finding the local optimum with accuracy. In the PSO schemes, a particle cannot reach any point in problem space quickly. In theory, since particles survive intact from one iteration to the next, any particle can eventually go anywhere. This might be possible at the beginning of the run but appears more difficult later on when approaching convergence, as the process is partially blocked. This issue can be treated via a strong 
cooperation with the mechanisms introduced in our AGAs. Stagnation has to be detected and the process relaunched via a refreshing process. It is however important not only to detect premature convergence but also to prevent it. The idea is to maintain as far as possible a population presenting a minimum of diversity so as to reinforce the probability of escaping from a local minimum. Redundant particles do not contribute further to the improvement of convergence. Thus, prevention can be achieved by continuously inspecting the presence of redundant particles and replacing some of them by new particles via a dedicated operator as done in our AGA.

Furthermore, strong cooperation between the PSO mechanism for exploration and AGA is likely to improve AGA performances, especially the runtime. Exploration is performed differently, generally quickly and this difference can be advantageously exploited.

After having underlined some obvious complementarities, it is worth mentioning several analogies between the algorithms.

Adapting the mutation in GA that has proved to be efficient is analogous to controlling the inertia weight (from rather large to small) as in NPSO and CPSO. Both parameters remain important. Their setting needs to be calibrated; they vary with the evolutionary life and are devoted to a specific chromosome or particle. Furthermore, the limitation is similar: as pointed out by Eberhart et al. [11], when the population is close to convergence and the average fitness value is high, mutation will quite likely result in a low-fitness chromosome that does not survive the selection process. So even though a number of mutations would bring the chromosome into a high-fitness region, the chromosome never gets there because it doesn't survive selection.

The crossover operation in GA can be partially retrieved in the different equations managing the particles' evolution. The analogy here is less obvious except that we can say that the exchange between elements remains static and not optimized in the basic version. Some sophisticated crossover schemes embed more intelligence and are analogous to PSO evolution especially if the crossover operation is enlarged to more than two chromosomes. In PSO, the notion of selectivity is indirectly present even if all the particles continue as members of the population for the duration of the run. In our opinion, the weakness is more due to the lack of control of selectivity which is better managed via AGA. Our ranking selection strategy maintains the best chromosomes but can eliminate the redundant ones to make room for more diversity and increase the chances of finding a better zone of the space. Unsurprisingly, we observe that the average accuracy clearly increases with the number of iterations, for all the methods, and that the relative order of the different methods does not strongly depend on the number of samples. While it is impossible to extrapolate the results to all the class of problems, it is not hard to imagine that PSO approaches could be more improved by embedding this intelligence as done for AGA. Hybridization [32] between the schemes is an interesting direction but needs to be well managed. We have implemented a preliminary hybrid scheme in the context of this study. This example has been processed to emphasize the power and to illustrate the improvement offered by the hybridization of PSO with the AGA. The idea is to use the ability of NPSO to quickly reach interesting zones of the search space and exploit them through GA. The algorithm starts the search for optimal solutions with the PSO to accelerate the exploration stage. Then, the AGA is set to fully exploit the space of solutions and so avoid local minima. With this basic hybridization version (AGA chromosomes initialized by NPSO), similar results as with AGA have been obtained while simultaneously reducing the runtime of AGA. The results (Fig. 9) show that the hybrid algorithm requires fewer iterations than the AGA algorithm for the optimization problem while maintaining the results (errors) comparable to those obtained by the AGA which are a better basis than those obtained by other methods mentioned in this paper. The runtime of the hybrid algorithm has been significantly reduced compared to the AGA because as mentioned earlier, 100 iterations in PSO requires only 3.44s. In future versions we envisage using NPSO each time the refreshing process is activated. We are also considering a stronger cooperation by managing the evolution of different subpopulations by exploiting several combinations of PSO and GA operators. The idea behind this cooperation is originally to optimize the search via the combination of mechanisms. Furthermore, it allows a niching strategy to be introduced: if the sub-populations can be mixed at different levels, they explore the search space in parallel, thereby increasing the probability of optimum by finding multiple solutions. 


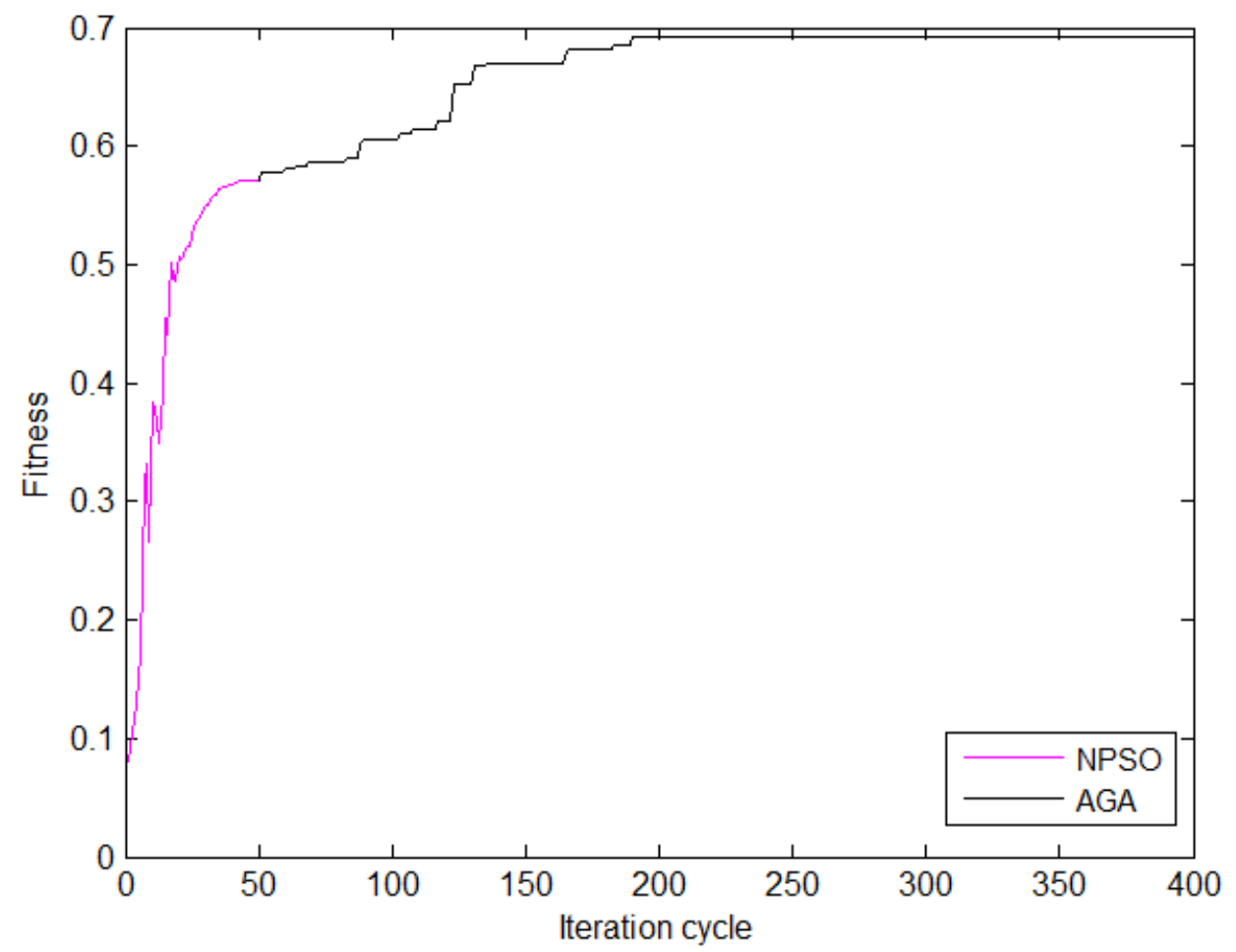

Fig. 9 Evolution of the fitness function in the hybrid algorithm in case of $40^{\text {th }}$ order Low Pass FIR filters $\left(\omega_{c}=0.5\right)$

\section{Conclusion}

In this paper, the standard genetic algorithm, adaptive genetic algorithm, particle swarm optimization, novel particle swarm optimization and craziness particle swarm optimization techniques have been applied to the design of FIR filters. An attractive advantage of PSO is the ease of implementation in both the context of coding and parameter selection. The algorithm is much simpler and intuitive to implement than the complex, probability-based selection and mutation operators required for evolutionary algorithms such as the GA. An advantage of the improved PSO is the low number of iterations required for convergence and again its ease of implementation. This study shows that CPSO can converge to the best quality near optimal solution and possesses the best convergence characteristics in much shorter runtimes than the GA, PSO and NPSO algorithms.

However, the results show that the speciation mechanisms implemented in AGA enable the algorithm to outperform the other ones. Our AGA may therefore be used as a good optimizer to obtain the optimal filter coefficients in a practical digital filter design problem in digital signal processing systems.

A significant difference between sophisticated versions of PSO and our AGA lies in the level of embedded intelligence. All of them have a layer that allows diversification but AGA goes further in continuously analyzing the evolution state and repeatedly self-tuning itself. It is also evident from the results obtained by a large number of trials that our AGA is consistently free from the shortcoming of premature convergence exhibited by the other optimization techniques.

We think that there is the potential to improve the PSO versions by embedding more intelligent mechanisms as has been done for AGA. Hybridization between the schemes is a promising field that has proved its ability to improve outcomes. It needs however to be well managed. Hybridization does not always perform as per expectations (lack of result repeatability, flexibility...). The improper use of mechanisms can waste algorithm resources and even lead to worse performances. Implementation of hybrid schemes is still an issue. Today, it should be said that this highlights an essential limitation of the use of hybridization but encourages the researchers working on this open field to develop more efficient hybrid models. Furthermore, it seems possible to challenge 
the researchers to unify the concepts behind PSO and GA: better cooperation between chromosomes in GA, better selectivity and capacity of diversification in PSO and for both, a better analysis of the evolution state to intelligently adapt the embedded mechanisms.

\section{References}

1. J. S. Lim, A. V. Oppenheim: Advanced topics in signal processing. Prentice-Hall (1988)

2. Z. Milivojevic: Digital filter design, mikroElektronika - 1st edition OnLine. http://www.mikroe.com/chapters/view/72/\#id22 (2007)

3. J. H. Holland: Genetic algorithms and the optimal allocation of trials. SIAM Journal on Computing 2, 88-105 (1973)

4. J. Kennedy, R. Eberhart: Particle Swarm Optimization. IEEE int. Conf. On Neural Network, vol. 04, 19421948 (1995)

5. R. C. Eberhart, Y. Shi: Particle swarm optimization: developments, applications and resources. Proceedings of IEEE Congress on Evolutionary Computation 2001 IEEE service center (2001)

6. R. C. Eberhart, Y. Shi: Tracking and optimizing dynamic systems with particle swarms. Proceedings of IEEE Congress on Evolutionary Computation 2001, IEEE service center, 94-97 (2001)

7. H. Liu, A. Abraham, W. Zhang: A fuzzy adaptive turbulent particle swarm optimization. International Journal of Innovative Computing and Applications 1, 39-47 (2007)

8. D. W. Van Der Merwe, A. P. Engelbrecht: Data clustering using particle swarm optimization. The 2003 Congress on Evolutionary Computation CEC'03, vol. 01, 215-220 (2003)

9. J. Robinson, S. Sinton, Y.R. Samii: Particle swarm, genetic algorithm, and their hybrids: optimization of a profiled corrugated horn antenna. Proceedings of the IEEE International Symposium in Antennas and Propagation Society, 314-317 (2002)

10. Gandelli, F. Grimaccia, M. Mussetta, P. Pirinoli, R.E. Zich: Genetical swarm optimization: an evolutionary algorithm for antenna design. Journal of Automatika 47 (3-4), 105-112 (2006)

11. R. C. Eberhart, Y. Shi: Comparison between Genetic Algorithms and Particle Swarm Optimization. Proc. 7th Int. Conf. on Evolutionary Computation, San Diego (2000)

12. W.-D. Chang, D.-M. Chang: Design of a higher-order digital differentiator using a particle swarm optimization approach. Mechanical Systems and Signal Processing 22, 233-247 (2008)

13. W.-D. Chang: Two-dimensional fractional-order digital differentiator design by using differential evolution algorithm. Digital Signal Processing 19, 660-667 (2009)

14. R. Thangaraj, M. Pant, A. Abraham, P. Bouvry: Particle swarm optimization: Hybridization perspectives and experimental illustrations. Appl. Math. Comput. (2011). doi:10.1016/j.amc.2010.12.053

15. J. I. Ababneh, M. H. Bataineh: Linear phase FIR filter design using particle swarm optimization and genetic algorithms. Digital Signal Processing 18, No. 4, 657-668 (2008)

16. S. Mondal, Vasundhara, R. Kar, D. Mandal, S. P. Ghoshal: Linear Phase High Pass FIR Filter Design using Improved Particle Swarm Optimization. World Academy of Science, Engineering and Technology 60, 16201627 (2011) 
17. R. Kar, D. Mandal, D. Roy, S. P. Ghoshal: FIR Filter Design using Particle Swarm Optimization with Constriction Factor and Inertia Weight Approach. ACEEE Int. J. on Electrical and Power Engineering, Vol. 02, No. 02, 1-5 (2011)

18. S. Mondal, S. P. Ghoshal, R. Kar, D. Mandal: Novel Particle Swarm Optimization for Low Pass FIR Filter Design. Wseas Transactions On Signal Processing, Issue 3, Vol. 08, 111-120 (2012)

19. R. Kar, D. Mandal, S. Mondal, S. P. Ghoshal: Craziness based Particle Swarm Optimization algorithm for FIR band stop filter design. Swarm and Evolutionary Computation 7, 58-64 (2012)

20. C. R. Reeves: A Genetic Algorithm For Flowshop Sequencing. Computers and Operations Research 22 (1), 5-13 (1995)

21. D. E. Goldberg: Genetic Algorithms in Search, Optimization, and Machine Learning. Addison-Wesley, Reading, MA (1989)

22. H. C. Lu, S. T. Tzeng: Design of arbitrary FIR log filters by genetic algorithm approach. Signal Processing 80 , 497-505 (2000)

23. S. T. Tzeng, H. C. Lu: Complex genetic algorithm approach for designing equiripple complex FIR digital filters with weighting function. Signal Processing 80, 197-204 (2000)

24. O. Franzen, H. Blume, H. Schroder: FIR-filter design with spatial and frequency design constraints using evolution strategies. Signal Processing 68, 295-306 (1998)

25. L. Cen: A hybrid genetic algorithm for the design of FIR filters with SPoT coefficients. Signal Processing 87 , 528-540 (2007)

26. J. He, H. Guo: A Modified Particle Swarm Optimization Algorithm. Telkomnika, Vol. 11, No. 10, 6209-6215 (2013)

27. D. Parrott, X. Li: Locating and Tracking Multiple Dynamic Optima by a Particle Swarm Model Using Speciation. IEEE Transactions on Evolutionary computation 10(4), (2006)

28. M. Neshat, G. Sepidnam, M. Sargolzaei, A. N. Toosi: Artificial fish swarm algorithm: a survey of the state-ofthe-art, hybridization, combinatorial and indicative applications. Artificial Intelligence Review (2012)

29. E. Pérez, M. Posada, F. Herrera: Analysis of new niching genetic algorithms for finding multiple solutions in the job shop scheduling. J. Intel. Manuf. 23, No. 03, 341-356 (2012)

30. K. Boudjelaba, D. Chikouche, F. Ros: Evolutionary techniques for the synthesis of 2-D FIR filters. 2011 IEEE Workshop on Statistical Signal Processing (SSP'11), 601-604 (2011)

31. K. Boudjelaba, F. Ros, D. Chikouche: An advanced genetic algorithm for designing 2-D FIR filters, 2011 IEEE Pacific Rim Conference on Communications, Computers and Signal Processing, 60-65 (2011)

32. F. Ros, S. Guillaume: An efficient nearest classifier, Book Chapter of Hybrid Evolutionary Systems. Studies in Computational Intelligence, Vol. 75, Springer Verlag, pp. 131-147 (2007)

33. D. Suckley: Genetic algorithm in the design of FIR filters. IEE Proceedings G, Circuits, Devices and Systems 138(2), 234-238 (1991)

34. N. E. Mastorakis, I. F. Gonos, M. N. S. Swamy: Design of two-dimensional recursive filters using genetic algorithms. IEEE Transactions On Circuits and Systems I: Fundamental Theory And Applications 50(5), 634-639 (2003) 
35. L. Zhao, L. Zhou, W. Huang: Satisfactory Optimization Design Of FIR Digital Filter Based On Adaptive Particle Swarm Optimization. IEEE International Conference on Control and Automation, 1662-1666 (2007)

36. M. G.H. Omran, A. P. Engelbrecht, A. Salman: Differential Evolution Based Particle Swarm Optimization. IEEE Swarm Intelligence Symposium, 112-119 (2007)

37. S. Jun, L. Jian: A Hybrid of Differential Evolution and Particle Swarm Optimization for Global Optimization. Third International Symposium on Intelligent Information Technology Application IITA 2009, 138-141 (2009)

38. B. Luitel, G.K. Venayagamoorthy: Differential Evolution Particle Swarm Optimization for Digital Filter Design. IEEE Congress on Evolutionary Computation (CEC 2008), 3954-3961 (2008)

39. A. Kaur, R. Kaur: Design of FIR Filter Using Particle Swarm Optimization Algorithm for Audio Processing. International Journal of Computer Science and Network (IJCSN), Volume 1, Issue 4, 103-108 (2012)

40. N. Karaboga, B. Cetinkaya: Design of digital fir filters using differential evolution algorithm. Circuits Systems Signal Processing 25(5), 649-660 (2006) 